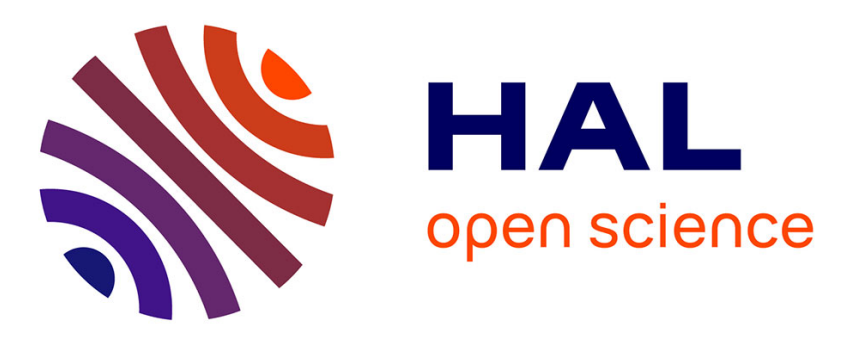

\title{
Effect of Surface Elasticity on the Rheology of Nanometric Liquids
}

Richard Villey, Emmanuelle Martinot, Cécile Cottin-Bizonne, Magali

Phaner-Goutorbe, Liliane Léger, Frédéric Restagno, Elisabeth Charlaix

\section{- To cite this version:}

Richard Villey, Emmanuelle Martinot, Cécile Cottin-Bizonne, Magali Phaner-Goutorbe, Liliane Léger, et al.. Effect of Surface Elasticity on the Rheology of Nanometric Liquids. Physical Review Letters, 2013, 111 (21), pp.215701. 10.1103/PhysRevLett.111.215701 . hal-00967566

\section{HAL Id: hal-00967566 https://hal.science/hal-00967566}

Submitted on 29 Mar 2014

HAL is a multi-disciplinary open access archive for the deposit and dissemination of scientific research documents, whether they are published or not. The documents may come from teaching and research institutions in France or abroad, or from public or private research centers.
L'archive ouverte pluridisciplinaire HAL, est destinée au dépôt et à la diffusion de documents scientifiques de niveau recherche, publiés ou non, émanant des établissements d'enseignement et de recherche français ou étrangers, des laboratoires publics ou privés. 


\title{
Effect of surface elasticity on the rheology of nanometric liquids.
}

\author{
Richard Villey, ${ }^{1}$ Emmanuelle Martinot, ${ }^{2}$ Cécile Cottin-Bizonne, ${ }^{1}$ Magali \\ Phaner-Goutorbe ${ }^{3}$ Liliane Léger, ${ }^{2}$ Frédéric Restagno, ${ }^{2, *}$ and Elisabeth Charlaix ${ }^{4}$ \\ 1 Institut Lumière Matière, Université Lyon 1 - CNRS, F-69622 Villeurbanne, France \\ ${ }^{2}$ Laboratoire de physique des solides, Université Paris-Sud-CNRS , F-91405 Orsay, France \\ 3 Institut des Nanotechnologies de Lyon, École Centrale de Lyon, \\ CNRS, Université de Lyon, F-69134 Ecully, France \\ ${ }^{4}$ Laboratoire interdisciplinaire de physique, Université Grenoble 1, CNRS, F-38402 Grenoble, France ${ }^{\dagger}$
}

\begin{abstract}
The rheological properties of liquids confined to nanometer scales are important in many physical situations. In this paper we demonstrate that the long range elastic deformation of the confining surfaces must be taken into account when considering the rheology of nanometric liquids. In the case of a squeeze-flow geometry, we show that below a critical distance Dc the liquid is clamped by its viscosity and its intrinsic properties cannot be disentangled from the global system response. Using nanorheology experiments, we demonstrate that picometer elastic deflections of the rigid confining surfaces dominate the overall mechanical response of nanometric liquids confined between solid walls.
\end{abstract}

The rheological properties of thin liquid films are of paramount importance in many fields such as the rheology of complex fluids and dispersed media, the flow of liquids in rocks and nanopores, the lubrication of rubbing solid contacts, nanofluidic devices and their applications. As a result the investigation of liquids confined at the nanometric scale has been an active field for many years, from the pionneering experiments of Chan and Horn in 1985 [1] up to the very recent investigations with the Atomic Force Microscope [2-6].

While the structure and physico-chemistry of the confining surfaces are important when considering the properties of confined liquids, their elastic behavior is usually not taken into account, as noted by E.J. Hinch [7] or later by O.I. Vinogradova [8]. Investigations of flows in thin liquid films are conducted in a conceptual framework where the long range deformations of the confining surfaces are either negligible, or can be routinely substracted, so that the intrinsic flow behaviour of the nanometric film is accessed.

In soft surfaces it is recognized that the coupling of elastic deformations and thin film flows leads to very nonintuitive effects. An example is the "dimple" that arises when a drop or bubble approaches another surface, forcing a draining flow. This can result in the formation of a "wimple"; a more complicated rippled shape [9]. Another example is droplets merging in a channel. Merging occurs when the droplets are pulled away from each other, not pushed together as might be expected [10]. In the case of hard solids, it has been recognized that elastohydrodynamic effects are important to understand the lubrication of solids [11] from nanometric contacts [12] to the lubrication of faults in geophysics [13].

In the present letter, we show that elastohydrodynamic effects are to be taken into account to understand the flow of confined liquids in nanometric confinement. More precisely, we show here that vanishingly small elastic deflections of the surfaces, of amplitude lower than the molecu- lar size, have an effect on the apparent rheology of nanometric films. The long-range character of elastic interactions does not allow to disentangle the confining bodies bulk deformations from the nanometric confined liquid flow.

We demonstrate quantitatively the effect of elastic couplings in the specific geometry of an oscillating drainage flow between a sphere and a plane, which is widely used to study thin films flows in particular with Surface forces apparatii (SFA) or Atomic force microscopes (AFM). Our demonstration is based on an exact elastohydrodynamic theory without ajustable parameters [14], and well-controlled experiments using very rigid surfaces (glass of Young modulus $E=64 \mathrm{GPa}$ ) and liquids of

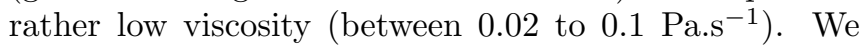
show that liquids confined below a critical thickness $D_{c}$ are clamped by their viscosity, while the surfaces accommodate for all the global displacements. We describe by "elastic confinement" this state in which it is not possible to access the rheology of the liquid film. The critical thickness for elastic confinement depends on the liquid and solid modulii, the rate, geometry, and type of flow, and can therefore widely vary for the same liquid. We illustrate values of $D_{c}$ between 5 to $15 \mathrm{~nm}$ in our experiments. Furthermore elastic effects lead to a spatial heterogeneity of the flow pattern. This effect and the associated effective stiffness of the surfaces, depend on the gap between the sphere and the plane, and cannot be calibrated out. We also show that even for film thicknesses much larger than $D_{c}$, elastic effects cannot be neglected, as surface indentations as small as a picometer have a measurable impact on the apparent rheological properties of the liquid. Finally we extend qualitatively the analysis of elastic effects to the case of flattened AFM tips.

We study here the drainage flow of four different Newtonian liquids with a dynamic surface force apparatus (SFA) [15] used as a nano-rheometer (fig. 1). The SFA 
(a)

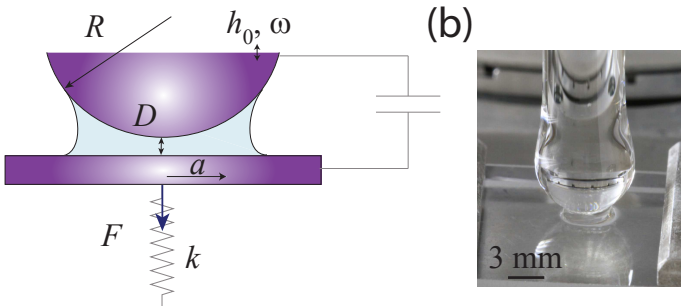

FIG. 1: (a) Principle of hydrodynamic impedance measurements: the relative distance $D(t)=D+h_{0} \cos \omega t$ between a millimetric sphere of radius $R$ and a plane is forced harmonically and its d.c. and a.c. components are measured with a capacitive sensor. The plane is mounted on a double spring cantilever of stiffness $k$ and the dynamic force $F(t)=F_{0} \cos (\omega t+\phi)$ is measured in amplitude and phase. The hydrodynamic impedance $Z^{*}(\omega, D)$ is the linear force response $F_{0} e^{i \phi} / h_{0}$. (b) Picture of the contact.

measures the relative distance $D(t)$ between a sphere and a plane, distance which is varied harmonically as $D(t)=D+h_{0} \cos \omega t$ in order to force an oscillatory drainage flow. In all following experiments the frequency is $\omega / 2 \pi=19 \mathrm{~Hz}$. The amplitude $h_{0}$ of the harmonic displacement is kept small $\left(h_{0}<0.1 D\right)$ in order to stay in the linear response domain. The SFA also measures the hydrodynamic impedance, defined as the complex force response $Z^{*}(\omega, D)=Z^{\prime}+i Z^{\prime \prime}=F^{*}(\omega) / h_{0}$ associated to the harmonic forcing, where $Z^{\prime}$ and $Z^{\prime \prime}$ are respectively the stiffness and the damping terms. In the case of a Newtonian liquid of viscosity $\eta_{0}$ confined between two rigid surfaces with a no-slip boundary condition, the hydrodynamic impedance is purely imaginary [16]:

$$
Z^{*}(\omega, D)=i Z^{\prime \prime}=i \frac{6 \pi \eta_{0} \omega R^{2}}{D}
$$

This expression has been widely used to study the rheology of liquid films between various surfaces [1, 16-19] and to test the boundary condition at the liquid-solid interface [20].

The liquids studied are two non-polar liquids: silicon oils $47 \mathrm{~V} 20\left(M_{w} \sim 3000 \mathrm{~g} / \mathrm{mol}, R_{g} \simeq 3 \mathrm{~nm}\right)$ and $47 \mathrm{~V} 100$ $\left(M_{w} \sim 11000 \mathrm{~g} / \mathrm{mol}, R_{g} \simeq 6 \mathrm{~nm}\right)$ purchased from Bluestar Silicones, and two polar liquids: glycerol-water mixtures made with analytical-grade glycerol (VWR analar, normapur). The bulk viscosities of the liquids range from 20 to $100 \mathrm{mPa} \cdot \mathrm{s}$ (tab. I). The confining solids are bulk borosilicate glass (pyrex, Schott). A sphere is prepared from a pyrex rod molten into a flame, washed in an ultrasonic bath with a detergent and ultra-clean water, and rinsed with purified propanol. The planes are $1 \mathrm{~cm} \mathrm{x} 1$ $\mathrm{cm}$ plates of borosilicate glass (thickness $5 \mathrm{~mm}$, Schott Borofloat 33, Young modulus $E=64 \mathrm{GPa}$ and Poisson ratio $\nu=0.2$ ) washed in the same way. The peak-topeak roughness measured by atomic force microscopy is
$0.5 \mathrm{~nm}$ over a $10 \mu \mathrm{m} \times 10 \mu \mathrm{m}$ area. The experiments are made at ambient temperature $T=25 \mp 1{ }^{\circ} \mathrm{C}$.

In figure 2, the stiffness $Z^{\prime}$ and the damping $Z^{\prime \prime}$ obtained with one of the glycerol-water mixtures are plotted as a function of $D$. Essentially two regimes can be observed. At large distances $(D>100 \mathrm{~nm})$, the damping decays as $1 / D$, as expected for the lubricating flow of a Newtonian liquid. This decay is in good agreement with eq. (1) using the bulk viscosity of the mixture. But a finite stiffness $Z^{\prime}$ is measured at any distance. This stiffness increases rapidly with the confinement. When $D<2 \mathrm{~nm}$, the stiffness becomes larger than the damping. This critical distance is the beginning of the second regime, in which both the stiffness and the damping saturate at a finite value.
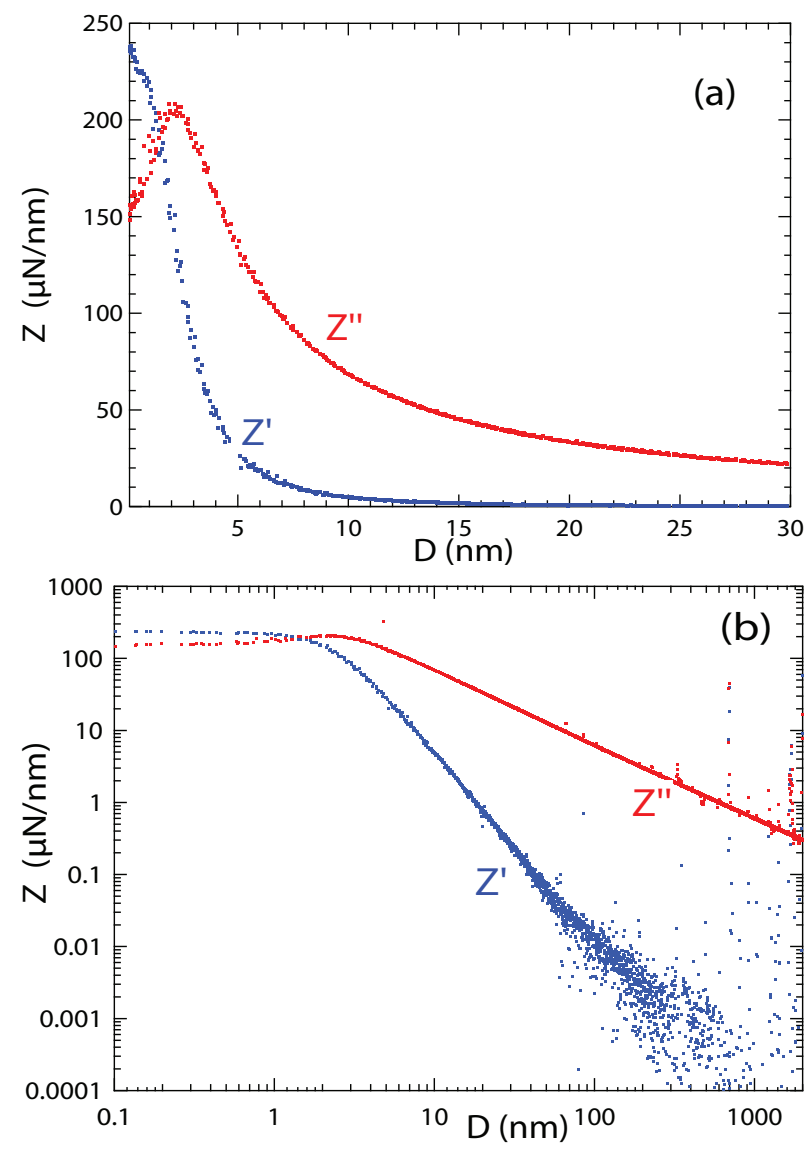

FIG. 2: Damping (red) and stiffness (blue) measured with the water-glycerol mixture of viscosity $24.9 \mathrm{mPa}$.s, in linear scale (a) and $\log -\log$ scale (b). The frequency is $\omega / 2 \pi=19 \mathrm{~Hz}$ and the radius of the sphere is $R=3.59 \mathrm{~mm}$.

A similar behavior is observed with the four studied liquids, which have quite different molecular properties and molecular sizes. This suggests that the rheological features plotted in fig. 2 may not be due to the liquid itself. Indeed, eq. (1) assumes perfectly rigid surfaces, which has no physical sense at small distances where the hydrodynamic force becomes very large. The drainage of a 
Newtonian liquid between a sphere and a plane is known to produce a pressure of the order of $P \simeq\left(3 \eta_{0} R / D^{2}\right) \dot{D}$ extending over a distance $\sqrt{2 R D}$ from the sphere-plane axis [1]. As a result, one can expect an elastic deflection $u$ of the confining surfaces of the order of $u / \sqrt{2 R D} \sim P / E^{*}$ with $E^{*}=E /\left(1-\nu^{2}\right)$ the reduced Young's modulus of glass and $\nu$ its Poisson ratio. The resulting deflection can be estimated as:

$$
u_{0} \sim 2 h_{0}\left(\frac{\omega \eta_{0}}{E^{*}}\right)\left(\frac{R}{D}\right)^{3 / 2}
$$

where the factor 2 accounts for both surfaces. For the system of figure 2, the order of magnitude of the deflection of the glass surface predicted by eq. (2) is of $16 \mathrm{pm}$ at $D=100 \mathrm{~nm}$ for $h_{0}=2 \mathrm{~nm}$, since the Young's modulus of the borosilicate glass is high. This deflection increases with the confinement, until it eventually reaches the amplitude of the forced oscillation $h_{0}[14,21]$. This transition is expected at a distance $D_{\mathrm{c}} \sim R\left(\omega \eta_{0} / E^{*}\right)^{2 / 3}$, in the range of $5-15 \mathrm{~nm}$ for liquids of viscosity between 10 to $100 \mathrm{mPa}$.s. This is the typical distance where major stiffness effects appear in fig. 2 and below which we can speak about elastic confinement.

\begin{tabular}{|l|l|l|l|l|}
\hline Fluid & $47 \mathrm{~V} 100$ & $47 \mathrm{~V} 20$ & $\begin{array}{l}\text { Glycerol- } \\
\text { water } \\
(\mathrm{a})\end{array}$ & $\begin{array}{l}\text { Glycerol- } \\
\text { water } \\
(\mathrm{b})\end{array}$ \\
\hline \hline$\eta_{0}(\mathrm{mPa} \cdot \mathrm{s})$ & $104.3 \pm 3$ & $22.9 \pm 0.6$ & $24.9 \pm 1$ & 35 \\
$R(\mathrm{~mm})$ & $3.37 \pm 0.04$ & $3.28 \pm 0.03$ & $3.25 \pm 0.04$ & $3.59 \pm 0.04$ \\
$D_{\mathrm{c}}(\mathrm{nm})$ & 14 & 5.0 & 5.2 & 7.2 \\
\hline
\end{tabular}

TABLE I: Experimental characteristics. The viscosity $\eta_{0}$ is obtained from the damping at large distance. $R$ is the sphere radius. $D_{\text {c }}$ is the cut-off distance defined in eq. (3), calculated using the Young's modulus $E=64 \mathrm{GPa}$ and the Poisson ratio $\nu=0.2$ of borosilicate glass (The Young's modulus that appear in eq. 3 has to be divided by 2 to take into account the deformation of the sphere and the plane. All experiments are performed at the frequency $\omega / 2 \pi=19 \mathrm{~Hz}$.

As a consequence, we expect that at distances of the order of $D_{c}$, the elastic deflection of glass reaches the amplitude of the forced oscillation. How does this affect the measured damping and stiffness ? The full elastohydrodynamic calculation of the oscillating drainage flow in the linear response limit, yields to the expression [14]:

$$
Z^{*}(\omega, D)=\frac{6 \pi \eta_{0} \omega R^{2}}{D_{\mathrm{c}}} g_{k}\left(\frac{D}{D_{\mathrm{c}}}\right) \quad D_{\mathrm{c}}=8 R\left(\frac{\omega \eta_{0}}{E^{*}}\right)^{2 / 3}
$$

where $g_{k}$ is a tabulated non-dimensional function not dependent on the liquid or the solid (numerical values of [14] are reported in the SI). In the limit $x \gg 1$, which is equivalent to $D \gg D_{\text {c }}$, the asymptotic value of $g_{k}$ allows to predict:

$$
Z^{*}(\omega, D)=\frac{6 \pi \eta_{0} \omega R^{2}}{D_{\mathrm{c}}}\left(\frac{9 \pi^{2}}{512}\left(\frac{D_{\mathrm{c}}}{D}\right)^{5 / 2}+i \frac{D_{\mathrm{c}}}{D}\right)
$$

The scaling prediction for the measured stiffness, $Z^{\prime} \sim$ $D^{-5 / 2}$, is characteristic of the elastic deformation of the two semi-infinite confining surfaces. It is indeed not possible to calibrate out this effect by assuming that the surfaces are equivalent to an internal stiffness of constant value $k$ : the force response of a spring-and-dashpot system of damping coefficient $\lambda$ is $i \lambda \omega /(1+i \lambda \omega / k) \simeq$ $i \lambda \omega+\lambda^{2} \omega^{2} / k$ for large $k$. With $\lambda=6 \pi \eta_{0} R^{2} / D$ for the Newtonian flow, this would give a decay $Z^{\prime} \sim D^{-2}$, different from the observed scaling. This is due to the fact that the actual surface stiffness is indeed distance dependant, and scales as the hydrodynamic radius $\sqrt{2 R D}$ of the Newtonian drainage flow. Thus the flow and the elastic effects are strongly coupled and cannot be simply disentangled from each other.

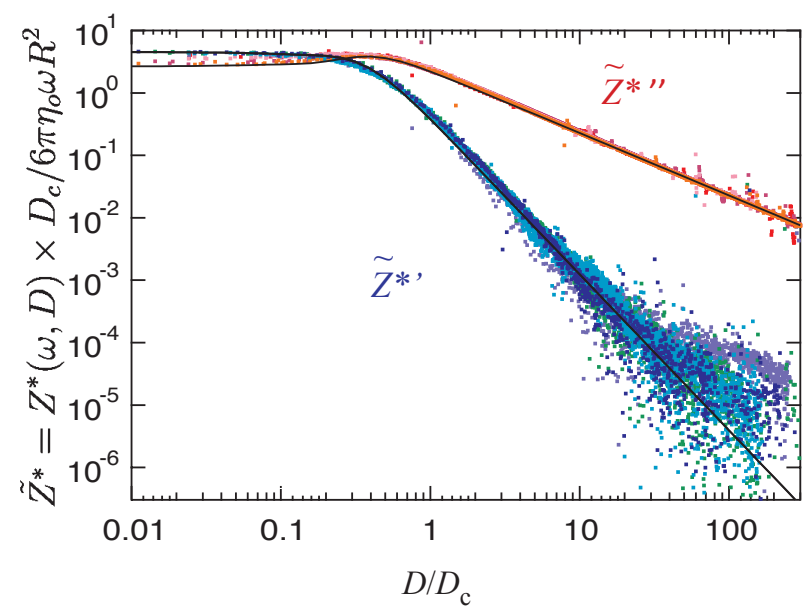

FIG. 3: Normalized impedance $\tilde{Z}^{*}=Z^{*}(\omega, D) \times D_{\text {c }} / 6 \pi \eta_{0} \omega R^{2}$ as a function of the normalized distance $D / D_{\text {c }}$. Data obtained with four different liquids are superimposed: two silicon oils, $47 \mathrm{~V} 20$ and 47V100, and two water-glycerol mixtures of different viscosities (see table I). $D_{c}$ is calculated in table I. The continuous lines are the real and imaginary parts of the complex master function $g_{k}(x)$ (see SI). The different blue and red points correspond to the four different liquids and can hardly be distinguished.

The elasto-hydrodynamic theory also predicts that

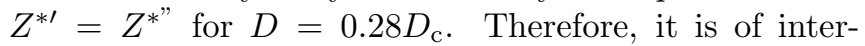
est to plot the reduced hydrodynamic impedance $\tilde{Z}^{*}=$ $Z^{*}(\omega, D) D_{\mathrm{c}} / 6 \pi \eta_{0} \omega R^{2}$ obtained for the four tested liquids, as a function of $D / D_{\text {c }}$. For each liquid, the critical distance $D_{c}$ is calculated using eq.(3) (see table I). The result is in fig. 3. All reduced impedances follow the same master curve, which is in quantitative agreement with the predicted function $g_{k}\left(D / D_{\mathrm{c}}\right)[14]$ without any adjustable parameter. This shows that the elastic deflection of glass is entirely responsible for the apparent deviation of the liquid response from the standard Newtonian behaviour.

In particular, the saturation of both the damping and the stiffness at $D<D_{c}$ reflects the spatial segregation of the flow. In a central region around the sphere-plane axis where the liquid thickness is less than $D_{c}$, the surfaces 
accomodate fully for the sphere displacement, and the liquid merely transmits the pressure to the surfaces. The usual lubrication flow occurs only in the outskirt of this central region where the liquid thickness is larger than $D_{c}$, and builds the pressure acting in the central region. As a consequence, at distances smaller than $D_{c}$, the stiffness and the damping only lightly reflect the intrinsic properties of the confined liquids.

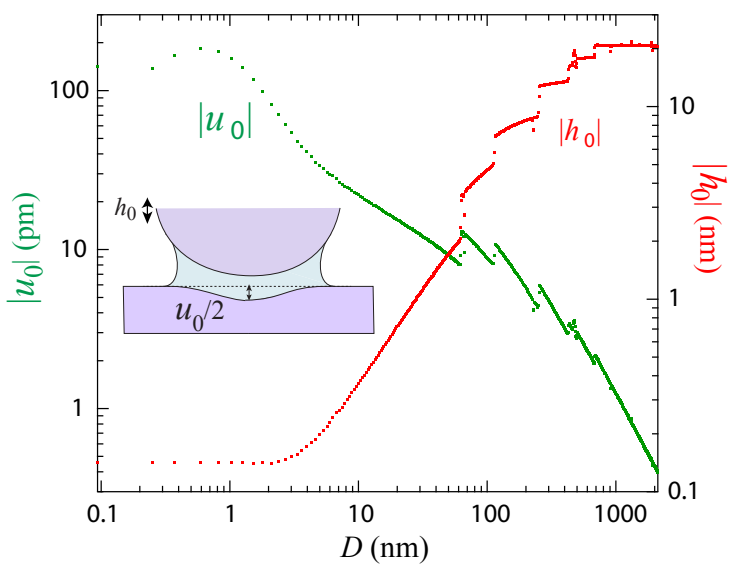

FIG. 4: The inset sketches the plane deformation due to the draining liquid. The same deformation occurs on the sphere (not sketched). In green, the amplitude of the glass surfaces indentation $\left|u_{0}\right|$ at the level of the sphere apex, calculated for the experiment with the water-glycerol (b) mixture plotted in fig. 2. In red, the corresponding amplitude $\left|h_{0}\right|$ of the forced oscillation is plotted on the right axis.

We have calculated more precisely (see SI) the glass surface deformation responsible for this apparent viscoelastic behavior. In fig. 4, the calculated amplitude $\left|u_{0}\right|$ of this deflection in the experiment presented in figure 2 is plotted as a function of the distance. The amplitude of the forced oscillation $h_{0}$ is varied by steps from $0.1 \mathrm{~nm}$ to $20 \mathrm{~nm}$. Consequently, the calculated indentation $\left|u_{0}\right|$ decreases by steps too, from $0.1 \mathrm{~nm}$ at $D=1 \mathrm{~nm}$ down to $1 \mathrm{pm}\left(10^{-12} \mathrm{~m}\right)$ at $D=1 \mu \mathrm{m}$. This is the order of magnitude calculated previously. This extremely small deflection corresponds to a relative change $\left|u_{0}\right| / h_{0}$ lower than $3.10^{-4}$ in the liquid film thickness. It is remarkable that such a small elastic deflection leads to a measurable effect in the dynamic response of the system, with a finite stiffness detected at distances larger than $100 \mathrm{~nm}$.

In conclusion, these results demonstrate in the case of a squeeze flow geometry, the strong influence of elastic couplings on the dynamics of confined liquids. We have defined a critical distance for elastic confinement, below which the liquid is clamped by its viscosity and its intrinsic rheological behaviour cannot be disentangled from the global system response. This does not mean that these intrinsic properties have changed. For the four liquids that we have studied, the global system response is fully described down to the nanometer scale by an exact elasto-hydrodnamic calculation without adjustable parameters.

Our results also show that the critical distance for elastic confinement depends strongly on the surfaces geometry. Taking the example of AFM for instance, which uses damping and stiffness measurements to characterize thin liquid films as in the present work [2-6], one may first estimate that for typical values of a probe radius of $100 \mathrm{~nm}$, liquid viscosity of 0.02 Pa.s (OMCTS), similar Young modulus as here and frequency of $1 \mathrm{kHz}$, the value $D c=2$ pm given by eq. (3) predicts negligible elastic effects. However, the actual shape of tips are seldom measured and they tend to flatten on repeated contacts. This is dramatic for expression (3), leading to an infinite critical distance. Extending the dimensional analysis to the (extreme) case of a flat disk of radius $R$ instead of a sphere, one has in this new geometry to balance the viscous damping $F_{v}=3 \pi \eta_{0} \omega R^{4} / 2 D^{3}$ with the surface stiffness $\pi R E^{*}$, in order to find a new critical distance $D_{c \text {,flat }}=8 R\left(\eta_{0} \omega / E^{*}\right)^{1 / 3}=1.2 \mathrm{~nm}$, not negligible considering that elastic couplings have a measurable impact above the critical distance.

Finally, elastic couplings have not often been taken into account in the studies of confined fluids, except for very soft surfaces such as tyres or bubbles [22, 23]. They deserve to be considered more systematically and could provide a thread to reconciliate apparently contradictory observations [2-6, 24-26]. However they depend strongly on the flow geometry and an adequate analysis remains to be done in the case of shear flow, which has been used in surface force balance studies. More generally they are of interest to understand better the mechanical properties of complex fluids and polymer matrix nano-composites, in which usual effective medium approaches should be modified when the distance between solid charges in less than the critical distance for elastic confinement.

We acknowledge the financial support of ANR program Merig.

* Electronic address: frederic.restagno@u-psud.fr

$\dagger$ Electronic address: elisabeth.charlaix@ ujf-grenoble.fr

[1] D. Chan and R. Horn, J. Chem 83, 5311 (1985).

[2] A. Maali, T. Cohen-Bouhacina, G. Couturier, and J. Aime, Phys. Rev. Lett. 96, 086105 (2006).

[3] T.-D. Li and E. Riedo, Phys. Rev. Letters 100 (2008).

[4] S. de Beer, D. van den Ende, and F. Mugele, Nanotechnology 21, 325703 (2010).

[5] S. H. Khan, G. Matei, S. Patil, and P. M. Hoffmann, Physical review letters 105, 106101 (2010).

[6] A. Labuda, K. Kobayashi, K. Suzuki, H. Yamada, and P. Grütter, Phys. Rev. Lett. 110, 066102 (2013).

[7] R. H. Davis, J.-M. Serayssol, and E. Hinch, Journal of Fluid Mechanics 163, 479 (1986).

[8] O. I. Vinogradova and F. Feuillebois, Langmuir 18, 5126 
(2002).

[9] V. S. Ajaev, R. Tsekov, and O. I. Vinogradova, Physics of Fluids 19, 061702 (2007).

[10] N. Bremond, A. R. Thiam, and J. Bibette, Physical review letters 100, 024501 (2008).

[11] D. Dowson and G. Higginson, Elastohydrodynamic lubrication (Pergamon Press, London, 1966).

[12] J. Gao, W. Luetdke, and U. Landman, Science 270, 605 (1995).

[13] E. E. Brodsky and H. Kanamori, Journal of Geophysical Research: Solid Earth (1978-2012) 106, 16357 (2001).

[14] S. Leroy and E. Charlaix, J. Fluid Mech. 674, 389 (2011).

[15] F. Restagno, J. Crassous, E. Charlaix, C. CottinBizonne, and M. Monchanin, Rev. Sci. Instruments 73, 2292 (2002).

[16] J.-M. Georges, S. Millot, J.-L. Loubet, and A. Tonck, J. Chem. Phys. 98, 7345 (1993).

[17] S. Granick, Science 253, 1374 (1991).

[18] J. Klein, Y. Kamiyama, H. Yoshizawa, J. N. Israelachvili,
G. H. Fredrickson, P. Pincus, and L. J. Fetters, Macromolecules 26, 5552 (1993).

[19] H.-J. Butt, B. Cappella, and M. Kappl, Surface science reports 59, 1 (2005).

[20] C. Neto, D. R. Evans, E. Bonaccurso, H. J. Butt, and V. S. J. Craig, Reports On Progress In Physics 68, 2859 (2005).

[21] S. Leroy, A. Steinberger, C. Cottin-Bizonne, F. Restagno, L. Léger, and E. Charlaix, Phys. Rev. Lett. 108, 264501 (2012).

[22] B. Persson, Sliding Friction (Springer, Berlin, 2000).

[23] A. Steinberger, C. Cottin-Bizonne, P. Kleimann, and E. Charlaix, Phys. Rev. Lett. 100 (2008).

[24] J. Klein and E. Kumacheva, The Journal of chemical physics 108, 6996 (1998).

[25] A. Demirel and S. Granick, Phys. Rev. Lett. 77, 2261 (1996).

[26] L. Bureau, Phys. Rev. Let. 104, 218302 (2010). 